\title{
EXISTENCE OF LIPSCHITZ AND SEMICONCAVE CONTROL-LYAPUNOV FUNCTIONS
}

\author{
LUDOVIC RIFFORD
}

\begin{abstract}
Given a locally Lipschitz control system which is globally asymptotically controllable to the origin, we construct a control-Lyapunov function for the system which is Lipschitz on bounded sets and we deduce the existence of another one which is semiconcave (and so locally Lipschitz) outside the origin. The proof relies on value functions and nonsmooth calculus.
\end{abstract}

\section{INTRODUCTION}

This paper is concerned with the stabilization problem for a standard control system of the form $\dot{x}(t)=f(x(t), u(t))$. Lyapunov-like techniques have been successfully used in many problems in control theory, such as stabilizability, asymptotic controllability and stability. Stabilization by smooth feedback has been a subject of research by many authors. Among them, Artstein provided an important contribution (see [3]), proving that the control system admits a smooth Lyapunov function if and only if there is a stabilizing relaxed feedback. Moreover, if the system is affine in the control, it is further the case that there exists an ordinary stabilizing feedback continuous outside the origin. In general however such a feedback fails to exist, as pointed out by Sontag and Sussmann [24] and by Brockett [8] among others $([22],[12])$. Consequently, the existence of a smooth Lyapunov function fails in general. This fact leads to the design of time-varying (see [15],[16]) or discontinuous feedbacks. The construction of the latter (see [11]) has used the existence of a locally Lipschitz control-Lyapunov function whose decrease condition is stated in terms of Dini derivates or equivalently of proximal subgradients. The first result of this article is that, under certain mild assumptions on $f$ (a local Lipschitz condition and bounded dynamics near the origin), for Globally Asymptotically Controllable systems, such controlLyapunov functions always exist. This fact extends the well-known result of Sontag [23] and brings an affirmative answer to a conjecture that has been attributed to Sontag and Sussmann. Furthermore, the main result shows that a semiconcave control-Lyapunov function outside the origin always exists under the same assumptions. The semiconcavity is an intermediate property between Lipschitz continuity and continuous differentiability. Semiconcave functions have been used for instance to obtain uniqueness results for weak

1991 Mathematics Subject Classification. 93D05, 93D20, 93B05, 34D20, 49J52, 49L25, $70 \mathrm{~K} 15$.

Key words and phrases. Asymptotic stabilizability, semiconcave Lyapunov function, nonsmooth analysis, viscosity solutions.

L. Rifford: Institut Girard Desargues, Université Claude Bernard Lyon I 69622 Villeurbanne, France. email: rifford@desargues.univ-lyon1.fr. 
solutions of Hamilton-Jacobi equations, see [19],[20]. More recently, attention has been focused on the differential properties of such functions, see [1], [2]. This semiconcavity will be exploited in forthcoming work to construct stabilizing feedbacks having certain regularity properties. Some works and general references related to this article include $[5,6,17,18,21]$.

\section{Definitions And STATEMENts of the RESUlts}

In this paper, we study systems of the general form

$$
\dot{x}(t)=f(x(t), u(t))
$$

where the state $x(t)$ takes values in a Euclidian space $\mathbb{X}=\mathbb{R}^{n}$, the control $u(t)$ takes values in a given set $U$, and $f$ satisfies the following hypotheses:

Assumption 2.1. $f$ is locally Lipschitz in $x$ (uniformly in $u$ ). That is, for all $x \in \mathbb{X}$, there exists $\mathcal{V}_{x}$ a neighborhood of $x$ and $L_{x} \geq 0$ such that

$$
\left\|f\left(y^{\prime}, u\right)-f(y, u)\right\| \leq L_{x}\left\|y^{\prime}-y\right\| \quad \forall y, y^{\prime} \in \mathcal{V}_{x}, \forall u \in U .
$$

Assumption 2.2. $f$ is bounded on the ball $R \bar{B} \times \mathcal{U}$ for all $R>0$ (or equivalently, in view of the preceding assumption for some $R>0$.)

A special element " 0 " is distinguished in $U$, and the state $x=0$ of $\mathbb{X}$ is an equilibrium point, i.e., $f(0,0)=0$ (No linear structure on $U$ is used, however). The set of admissible controls is the set of measurable and locally essentially bounded functions $u: \mathbb{R}_{>0} \longrightarrow U . \mathbb{R}_{>0}$ denotes nonnegative reals, $B$ the open ball $B(0,1):=\{x:\|x\|<1\}$ in $\mathbb{X}$ and $\bar{B}$ the closure of $B$. We now introduce our definitions and the main result.

Definition 2.3. The system (2.1) is Globally Asymptotically Controllable (abbreviated GAC) if there exist a nonincreasing function $M: \mathbb{R}_{>0} \longrightarrow \mathbb{R}_{>0}$ such that $\lim _{R \downarrow 0} M(R)=0$ and a function $T: \mathbb{R}_{>0} \times \mathbb{R}_{>0} \longrightarrow \mathbb{R}_{\geq 0}$ with the following property:

For any $0<r<R$, for each $\|\xi\| \leq R$, there exist a control $u: \mathbb{R}_{\geq 0} \longrightarrow U$ and corresponding trajectory $x(\cdot): \mathbb{R}_{\geq 0} \longrightarrow \mathbb{X}$ such that

1) $\lim _{t \rightarrow \infty} x(t)=0$;

2) $\forall t \geq 0,\|x(t)\| \leq M(R)$

3) $\forall t \geq T(r, R),\|x(t)\| \leq r$.

REMARK 2.4. A routine argument involving continuity of trajectories with respect to initial states shows that the requirements of the above standard definition are equivalent to the following apparently weaker pair of conditions used in some references (see [25],[26]):

1) For each $\xi \in \mathbb{X}$ there is a control $u: \mathbb{R}_{\geq 0} \longrightarrow U$ that drives $\xi$ asymptotically to 0 ;

2) for each $\epsilon>0$, there is a $\delta>0$ such that for each $\xi \in \mathbb{X}$ with $\|\xi\| \leq \delta$ there is a control $u: \mathbb{R}_{\geq 0} \longrightarrow U$ that drives $\xi$ asymptotically to 0 and such that the corresponding trajectory $x(\cdot)$ satisfies $\|x(t)\| \leq \epsilon$ for all $t \geq 0$.

Moreover, the authors of [25],[26] add a condition on bounded controls; this one implies the assumption 2.2 by restriction on the system near the origin.

A function $V: X \longrightarrow \mathbb{R}_{\geq 0}$ is positive definite if $V(0)=0$ and $V(x)>0$ for $x \neq 0$, and proper if $V(x) \rightarrow \infty$ as $\|x\| \rightarrow \infty$.

IGD, , , 
Definition 2.5. A Lyapunov pair for the system (2.1) is a pair $(V, W)$ consisting of a continuous, positive definite, proper function $V: \mathbb{X} \longrightarrow \mathbb{R}$ and a positive definite continuous function $W: \mathbb{X} \longrightarrow \mathbb{R}$, with the property that for each $x \in \mathbb{X} \backslash\{0\}$ we have

$$
\forall \zeta \in \partial_{P} V(\xi), \inf _{u \in U}\langle\zeta, f(x, u)\rangle \leq-W(x) .
$$

Here $\partial_{P} V(x)$ refers to the proximal subdifferential of $V$ at $x$ (which may be empty): $\zeta$ belongs to $\partial_{P} V(x)$ iff there exists $\sigma$ and $\eta>0$ such that

$$
V(y)-V(x)+\sigma\|y-x\|^{2} \geq\langle\zeta, y-x\rangle \quad \forall y \in x+\eta B .
$$

The condition (2.2) is in fact equivalent to another one often used in the definition of nonsmooth Lyapunov function (see [23], [25], [26]); this other notion is based on the notion of directional or Dini subderivate. The equivalence between these two conditions is a consequence of Subbotin's Theorem (see for example [14], our principal source for the theory of nonsmooth analysis and [10] for a discussion of the equivalence). We remark that there exists a complete calculus of proximal subdifferentials, one that extends all the theorems of the usual smooth calculus.

Definition 2.6. A control-Lyapunov function (CLF) for the system (2.1) is a function $V: \mathbb{X} \longrightarrow \mathbb{R}$ such that there exists a continuous positive definite $W: \mathbb{X} \longrightarrow \mathbb{R}$ with the property that $(V, W)$ is a Lyapunov pair for (2.1).

We will say that $V$ is a locally Lipschitz control-Lyapunov function if $V$ is a control-Lyapunov function which is locally Lipschitz on $\mathbb{X}$. We claim the following theorem.

Theorem 2.7. Let $(f, U)$ be a control system as described above. Then under the assumptions 2.1 and 2.2, if the system is Globally Asymptotically Controllable, there exists a locally Lipschitz control-Lyapunov function.

REMARK 2.8. The converse is true and relatively easy if we suppose that $f$ is continuous in $u$ (we need this to obtain the existence of trajectories).

We now recall the definition of a semiconcave function [19] in an open set $\Omega$ of $\mathbb{X}$.

Definition 2.9. Let $u: \Omega \longrightarrow \mathbb{R}$ be a continuous function on $\Omega$; it is said to be semiconcave on $\Omega$ if for any point $x_{0} \in \Omega$ there exist $\rho, C>0$ such that

$$
u(x)+u(y)-2 u\left(\frac{x+y}{2}\right) \leq C\|x-y\|^{2},
$$

for all $x, y \in x_{0}+\rho B$.

We shall deduce as a corollary of the preceding theorem the main result of this article.

TheOREM 2.10. Let $(f, U)$ be a control system as described above. Then under the assumptions 2.1 and 2.2, if the system is Globally Asymptotically Controllable, there exists a continuous control-Lyapunov function semiconcave on $\mathbb{X} \backslash\{0\}$.

REMARK 2.11. The CLF is a viscosity supersolution of

$$
\sup _{u \in U}\{-\langle f(x, u), D V\}-W \geq 0 .
$$


We begin by giving some regularity results about certain value functions. Then we give the proof of Theorem 2.7. In the last section, we conclude with the proof of Theorem 2.10.

\section{A Result on Value Functions in finite time}

Throughout this section, we are given a multifunction $F$ mapping $\mathbb{X}$ to the subsets of $\mathbb{X}$, and we consider the differential inclusion

$$
\dot{x}(t) \in F(x(t)) \quad \text { a.e.. }
$$

A solution $x(\cdot)$ of $(3.1)$ on the interval $[a, b]$ is taken to mean an absolutely continuous function $x:[a, b] \longrightarrow \mathbb{X}$ which, together with $\dot{x}$, satisfies (3.1); such an arc will be called a $F$-trajectory on the interval $[a, b]$. We need for this section two properties of $F$ which turn out to be particulary important.

Assumption 3.1. The multifunction $F$ is locally Lipschitz with non-empty compact convex values.

Assumption 3.2. For some positive constants $K$ and $M$, and for all $x \in \mathbb{X}$,

$$
v \in F(x) \Longrightarrow\|v\| \leq K\|x\|+M
$$

(that is called the linear growth condition).

Under these two conditions, for all $x_{0} \in \mathbb{X}$, there exists a trajectory of (3.1) defined on $\mathbb{R}_{\geq 0}$ such that $x(0)=x_{0}$, and for any trajectory with initial data $x_{0}$ we have the following estimate

$$
\forall t \geq 0,\|x(t)\| \leq\left\|x_{0}\right\| e^{K t}+M t e^{K t} .
$$

This inequality is an easy consequence of Gronwall's Lemma (see [14]).

Let there be given a function $L: \mathbb{X} \longrightarrow \mathbb{R}_{\geq 0}$ and a compact set $\mathcal{T}$ of $\mathbb{X}$ satisfying

Assumption 3.3. $L$ is locally Lipschitz and for all $x \in \mathbb{X}, L(x) \geq 1$.

Assumption 3.4. There exists $\delta>0$ such that $\forall x \in \mathcal{T}, \delta \bar{B} \subset F(x)$.

We proceed now to define a value function $V(\cdot)$ on $\mathbb{X}$ in terms of trajectories of $F$ as follows:

$$
V(x):=\inf \left\{\int_{0}^{T} L(x(t)) d t: x(0)=x, \dot{x}(t) \in F(x(t)) \text { a.e. and } x(T) \in \mathcal{T}\right\} .
$$

(Note that $T$ is a choice variable in this "free-time" problem.)

We introduce the notation

$$
\mathcal{R}:=\{x \in \mathbb{X}: V(x)<+\infty\},
$$

the letter $\mathcal{R}$ stands for reachable: the set of points where $V$ is finite is the sets of points which can be driven to the target $\mathcal{T}$ in finite time. We have the following theorem.

Theorem 3.5. Assume (3.1)-(3.4). Then

(i) $\mathcal{R}$ is open;

(ii) $V$ is locally Lipschitz in $\mathcal{R}$;

(iii) $\forall x \in \mathcal{R} \backslash \mathcal{T}, \forall \zeta \in \partial_{P} V(x), \min _{v \in F(x)}\langle\zeta, v\rangle \leq-L(x)$. IGD, , , 
Proof. First, by the Lipschitz condition on $F$ and (3.4), there exists $0<$ $r \leq 1$ such that $\forall x \in \mathcal{T}+r \bar{B}, \frac{\delta}{2} \bar{B} \subset F(x)$. Hence, each state $x$ of $\mathcal{T}+r \bar{B}$ can be driven to $\mathcal{T}$ by a trajectory of $(3.1)$ in time $\frac{2}{\delta} d(x, \mathcal{T})$ (where $d(x, \mathcal{T})$ denotes $\left.\min _{\tau \in \mathcal{T}}\|x-\tau\|\right)$. This proves that $V$ is finite on $\mathcal{T}+r \bar{B}$, if we set $m:=\max _{x \in \mathcal{T}+r \bar{B}} L(x)$, we have

$$
\forall x \in \mathcal{T}+r \bar{B}, V(x) \leq \frac{2 m}{\delta} d(x, \mathcal{T}) .
$$

Fix now $x_{0} \notin \mathcal{T}$ such that $V\left(x_{0}\right)<+\infty$.

By the definition of $V$, there exists a $F$-trajectory $x_{0}(\cdot)$ and $T>0$ such that $x_{0}(0)=x_{0}, x_{0}(T) \in \mathcal{T}$ and

$$
\int_{0}^{T} L(x(s)) d s \leq V\left(x_{0}\right)+1 .
$$

The estimate (3.2) gives

$$
\forall t \in[0, T],\left\|x_{0}(t)\right\| \leq\left\|x_{0}\right\| e^{K T}+M T e^{K T} .
$$

Let $A:=\left[\left\|x_{0}\right\|+M T\right] e^{K T}$, let $\lambda_{F}$ the Lipschitz constant of $F$ on the ball $(A+1) \bar{B}$. Fix $y \in B\left(x_{0}, r e^{-\lambda_{F} T}\right)$.

By the corollary 1 p121 of [4], there exists a $F$-trajectory $y(\cdot)$ such that $y(0)=y$ and verifying

$$
\forall t \in[0, T],\left\|y(t)-x_{0}(t)\right\| \leq e^{\lambda_{F} T}\left\|y-x_{0}\right\|, \text { and }\|y(t)\| \leq A+1 .
$$

Consequently, if we set $\lambda_{L}$ the Lipschitz constant of $L$ on the ball $(A+1) \bar{B}$, we obtain

$$
\begin{aligned}
\int_{0}^{T} L(y(s)) d s & \leq \int_{0}^{T} L\left(x_{0}(s)\right) d s+\int_{0}^{T}\left[L(y(s))-L\left(x_{0}(s)\right)\right] d s \\
& \leq V\left(x_{0}\right)+1+\int_{0}^{T} \lambda_{L}\left\|y(s)-x_{0}(s)\right\| d s \\
& \leq V\left(x_{0}\right)+1+T \lambda_{L} e^{\lambda_{F} T}\left\|y-x_{0}\right\| \\
& \leq V\left(x_{0}\right)+1+T \lambda_{L} r .
\end{aligned}
$$

On the other hand, $d(y(T), \mathcal{T}) \leq\left\|y(T)-x_{0}(T)\right\| \leq e^{\lambda_{F} T}\left\|y-x_{0}\right\| \leq r$; this implies by (3.3) that

$$
V(y(T)) \leq \frac{2 m}{\delta} d(y(T), \mathcal{T}) \leq \frac{2 m r}{\delta} .
$$

Consequently, we have that for all $y \in B\left(x_{0}, r e^{-\lambda_{F} T}\right)$,

$$
\begin{aligned}
V(y) & \leq \int_{0}^{T} L(y(s)) d s+\frac{2 m r}{\delta} \\
& \leq V\left(x_{0}\right)+1+T \lambda_{L} r+\frac{2 m r}{\delta}=: c<+\infty .
\end{aligned}
$$

We have shown that $B\left(x_{0}, r e^{-\lambda_{F} T}\right) \subset \mathcal{R}$ which gives (i).

Now, let $x \in B\left(x_{0}, r e^{-\lambda_{F} T}\right)$, then for each positive integer $n$, there exists a $F$-trajectory $x_{n}(\cdot)$ and $T_{x}^{n} \geq 0$ such that $x_{n}(0)=x, x_{n}\left(T_{x}^{n}\right) \in \mathcal{T}$ and

$$
\int_{0}^{T_{x}^{n}} L\left(x_{n}(s)\right) d s \leq V(x)+\frac{1}{n} .
$$


Thus $L \geq 1$ implies $T_{x}^{n} \leq V(x)+\frac{1}{n} \leq c+\frac{1}{n}$ by (3.6). As before, the estimate (3.2) gives for each $n$

$$
\begin{aligned}
\forall t \in\left[0, T_{x}^{n}\right],\left\|x_{n}(t)\right\| & \leq\|x\| e^{K T_{x}^{n}}+M T_{x} e^{K T_{x}^{n}} \\
& \leq[\|x\|+M(c+1)] e^{K(c+1)} \\
& \leq\left[\left\|x_{0}\right\|+1+M(c+1)\right] e^{K(c+1)} .
\end{aligned}
$$

So we find a uniform bound for $\left\|\dot{x_{n}}(\cdot)\right\|$ on the intervals $\left[0, T_{x}^{n}\right] \subset[0, V(x)+1]$. Hence, the theorem of Arzela-Ascoli and the compactness of trajectories (see [14]) imply that there exists a trajectory $x(\cdot)$ with initial data $x$ such that $x\left(T_{x}\right) \in \mathcal{T}$ and

$$
V(x)=\int_{0}^{T_{x}} L(x(s)) d s
$$

with $T \leq V(x)$. That means that the infimum is attained in the definition of $V$.

We set $A^{\prime}:=\left[\left\|x_{0}\right\|+1+M(c+1)\right] e^{K(c+1)}$, and $\lambda_{F}^{\prime}$ the Lipschitz constant of $F$ on the ball $\left(A^{\prime}+1\right) \bar{B}$. We proceed to show that $V$ is Lipschitz on the ball $\bar{B}\left(x_{0}, \frac{r}{2} e^{-\lambda_{F}^{\prime}(c+1)}\right)$.

Fix $x, y$ in $\bar{B}\left(x_{0}, \frac{r}{2} e^{-\lambda_{F}^{\prime}(c+1)}\right)$. Then there exists as above $x(\cdot)$ a $F$-trajectory and $T_{x} \geq 0$ such that $x(0)=x, x\left(T_{x}\right) \in \mathcal{T}$ and

$$
V(x)=\int_{0}^{T_{x}} L(x(s)) d s .
$$

By $[4$, Cor.1,p.121], there exists a $F$-trajectory $y(\cdot)$ such that $y(0)=y$ and verifying

$$
\forall t \in\left[0, T_{x}\right],\|y(t)-x(t)\| \leq e^{\lambda_{F}^{\prime} T_{x}}\|y-x\| \text {, and }\|y(t)\| \leq A^{\prime}+1 .
$$

Consequently, if we set as before $\lambda_{L}^{\prime}$ the Lipschitz constant of $L$ on the ball $\left(A^{\prime}+1\right) \bar{B}$, we obtain

$$
\begin{aligned}
\int_{0}^{T_{x}} L(y(s)) d s & \leq \int_{0}^{T_{x}} L(x(s)) d s+\int_{0}^{T_{x}} \lambda_{L}^{\prime}\|y(s)-x(s)\| d s \\
& \leq V(x)+T_{x} \lambda_{L}^{\prime} e^{\lambda_{F}^{\prime} T_{x}}\|y-x\| \\
& \leq V(x)+c \lambda_{L}^{\prime} e^{\lambda_{F}^{\prime} c}\|y-x\|
\end{aligned}
$$

Now, $V\left(y\left(T_{x}\right)\right) \leq \frac{2 m}{\delta} d\left(y\left(T_{x}\right), \mathcal{T}\right) \leq \frac{2 m}{\delta} e^{\lambda_{F}^{\prime} c}\|y-x\|$. Hence, we conclude that

$$
V(y) \leq V(x)+\left[c \lambda_{L}^{\prime}+\frac{2 m}{\delta}\right] e^{\lambda_{F}^{\prime} c}\|y-x\| .
$$

Thus, because all the constants in the preceding inequality are independent of $x$ and $y$, we find

$$
|V(y)-V(x)| \leq\left[(c+1) \lambda_{L}^{\prime}+\frac{2 m}{\delta}\right] e^{\lambda_{F}^{\prime}(c+1)}\|y-x\|,
$$

which proves (ii). We now have to prove (iii). For that, consider $x \in \mathcal{R} \backslash \mathcal{T}$, and $x(\cdot)$ a trajectory of (3.1) for which the infimum of the definition of $V(x)$ is attained. Let $\zeta$ belonging to $\partial_{P} V(x)$, then there exists $\sigma$ and $\eta>0$ such that IGD, , ,

$$
V(y)-V(x)+\sigma\|y-x\|^{2} \geq\langle\zeta, y-x\rangle \quad \forall y \in x+\eta B .
$$


By optimality of the trajectory $x(\cdot)$, for all $t \in[0, T], V(x(t))=\int_{t}^{T} L(x(s)) d s$. Then, for $t$ sufficiently small,

$$
\int_{t}^{T} L(x(s)) d s-\int_{0}^{T} L(x(s)) d s+\sigma\|x(t)-x\|^{2} \geq\langle\zeta, x(t)-x\rangle,
$$

which gives

$$
-\frac{1}{t} \int_{0}^{t} L(x(s)) d s+t \sigma\left\|\frac{x(t)-x}{t}\right\|^{2} \geq\left\langle\zeta, \frac{x(t)-x}{t}\right\rangle .
$$

We find (iii) by passing to the limit: $t \downarrow 0$.

REMARK 3.6. In [10], a result of this type is proven differently by an appeal to Hamiltonian necessary conditions.

REMARK 3.7. The conclusions of Theorem 3.5 remain true if we weaken the assumption 3.4 to the proximal condition

$$
\min _{v \in F(x)}\langle\zeta, v\rangle \leq-\delta\|\zeta\|
$$

forall $x \in \mathcal{T}$ where $\zeta \in N_{\mathcal{T}}^{P}(x)$. (This result is a consequence of proximal criteria for attainability, see [13],[14].) This kind of condition added to the smooth regularity of $F$ is used in [9] to obtain the semiconcavity of the minimum-time function. However, these results (on the Lipschitz property or on the semiconcavity property) do not hold if we omit the linear growth condition (3.2); see for example [7, Ex.1.3,p.238]

REMARK 3.8. The conclusion (iii) can be strengthened to equality. The value function $V$ is the viscosity solution of a certain Hamilton-Jacobi equation (see [7],[14]).

\section{Proof of Theorem 2.7}

We suppose first that we have constructed a control-Lyapunov function $V$ which is continuous on $\mathbb{X}$ and locally Lipschitz on $\mathbb{X} \backslash\{0\}$. Thus, there exists another continuous positive definite function $W: \mathbb{X} \longrightarrow \mathbb{R}_{>0}$ such that $(V, W)$ is a Lyapunov pair for (2.1). We proceed to show that we can deduce the existence of a new control-Lyapunov function which is locally Lipschitz on all the space $\mathbb{X}$. We set for any $0 \leq a \leq b$

$$
S_{V}(b):=\{x ; V(x) \leq b\} \text { and } S_{V}[a, b]:=\{x ; a \leq V(x) \leq b\},
$$

they are compact sets of $\mathbb{X}$. We proceed to construct a sequence of functions on $\mathbb{X}$ which will converge uniformly to our desired locally Lipschitz controlLyapunov function.

First, we set $\mathcal{V}_{0}(x):=\max \{V(x), 1\}$. This function is locally Lipschitz on $\mathbb{X}$, proper, positive, constant on $S_{V}(1)$ and verifies

$$
\forall x \notin S_{V}(1), \forall \zeta \in \partial_{P} \mathcal{V}_{0}(x), \inf _{u \in \mathcal{U}}\langle\zeta, f(x, u)\rangle \leq-W(x) .
$$

By assumption, for all $n \geq 0, V$ is Lipschitz on $S_{V}\left[\frac{1}{2^{n+1}}, \frac{1}{2^{n}}\right]$, we note $K\left(\frac{1}{2^{n+1}}, \frac{1}{2^{n}}\right)$ its Lipschitz constant on this set (without lost of generality 
we can choose this constant smaller than 1 ).

We define now a sequence inductively; suppose $\mathcal{V}_{n}$ given, we set

$$
\mathcal{V}_{n+1}(x):= \begin{cases}\mathcal{V}_{n}(x) & \text { si } x \notin S_{V}\left(\frac{1}{2^{n}}\right) \\ \mathcal{V}_{n}(x)+\frac{1}{K\left(\frac{1}{2^{n+1}}, \frac{1}{2^{n}}\right)}\left[V(x)-\frac{1}{2^{n}}\right] & \text { si } x \in S_{V}\left[\frac{1}{2^{n+1}}, \frac{1}{2^{n}}\right] \\ \mathcal{V}_{n}(x)-\frac{1}{2^{n+1} K\left(\frac{1}{2^{n+1}}, \frac{1}{2^{n}}\right)} & \text { si } x \in S_{V}\left(\frac{1}{2^{n+1}}\right)\end{cases}
$$

We have the following lemma.

Lemma 4.1. For all $n \geq 1, \mathcal{V}_{n}$ is 1-Lipschitz on $S_{V}(1)$, proper, and constant on $S_{V}\left(\frac{1}{2^{n}}\right)$. Moreover, $\mathcal{V}_{n}$ satisfies the following properties:

$$
\forall x \in \mathbb{X}, \mathcal{V}_{n}(x) \geq 1-\sum_{k=1}^{n} \frac{1}{2^{k} K\left(\frac{1}{2^{k}}, \frac{1}{2^{k-1}}\right)}, \quad \text { and }
$$

$\forall x \in S_{V}\left(\frac{1}{2^{n-1}}\right) \backslash S_{V}\left(\frac{1}{2^{n}}\right), \forall \zeta \in \partial_{P} \mathcal{V}_{n}(x)$,

$$
\inf _{u \in \mathcal{U}}\langle\zeta, f(x, u)\rangle \leq-\frac{W(x)}{K\left(\frac{1}{2^{n}}, \frac{1}{2^{n-1}}\right)} .
$$

Proof. We're going to prove only the last assertion. The other ones are left to the reader, they are the consequence on an easy inductive proof.

Let $n \geq 1, x \in S_{V}\left(\frac{1}{2^{n-1}}\right) \backslash S_{V}\left(\frac{1}{2^{n}}\right)$, and $\forall \zeta \in \partial_{P} \mathcal{V}_{n}(x)$.

We remark that for all $y$ not in $S_{V}\left(\frac{1}{2^{n}}\right)$, we have

$$
\mathcal{V}_{n}(y)=\min \left\{\mathcal{V}_{n-1}(y), \mathcal{V}_{n-1}(y)+\frac{V(y)-\frac{1}{2^{n-1}}}{K\left(\frac{1}{2^{n}}, \frac{1}{2^{n-1}}\right)}\right\} \text {. }
$$

For $x$, the minimum is attained in the second term, so

$$
\zeta \in \partial_{P}\left[\mathcal{V}_{n-1}(x)+\frac{\mathcal{V}(x)-\frac{1}{2^{n-1}}}{K\left(\frac{1}{2^{n}}, \frac{1}{2^{n-1}}\right)}\right]=\partial_{P}\left[\mathcal{V}_{n-1}(x)+\frac{\mathcal{V}(x)}{K\left(\frac{1}{2^{n}}, \frac{1}{2^{n-1}}\right)}\right]
$$

First case: $n>1$. We remark now that $\forall y \in S_{V}\left(\frac{1}{2^{n-2}}\right)$,

$$
\mathcal{V}_{n-1}(x)=\max \left\{C_{n-2}+\frac{V(x)-\frac{1}{2^{n-2}}}{K\left(\frac{1}{2^{n-1}}, \frac{1}{2^{n-2}}\right)}, C_{n-2}-\frac{1}{2^{n-1} K\left(\frac{1}{2^{n-1}}, \frac{1}{2^{n-2}}\right)}\right\},
$$

where $C_{n-2}$ is the value of $\mathcal{V}_{n-2}$ on the set $S_{V}\left(\frac{1}{2^{n-2}}\right)$. We deduce by (4.2) that

$$
\zeta \in \partial_{P}\left[\max \left\{\frac{V(x)}{K\left(\frac{1}{2^{n-1}}, \frac{1}{2^{n-2}}\right)}+A, A^{\prime}\right\}+\frac{V(x)}{K\left(\frac{1}{2^{n}}, \frac{1}{2^{n-1}}\right)}\right] .
$$

where $A=C_{n-2}-\frac{1}{2^{n-2} K\left(\frac{1}{2^{n-1}}, \frac{1}{2^{n-2}}\right)}$ et $A^{\prime}=C_{n-2}-\frac{1}{2^{n-1} K\left(\frac{1}{2^{n-1}}, \frac{1}{2^{n-2}}\right)}$.

Hence, we obtain that $\zeta$ is in the set

$\partial_{P}\left[\max \left\{\left(\frac{1}{K\left(\frac{1}{2^{n-1}}, \frac{1}{2^{n-2}}\right)}+\frac{1}{K\left(\frac{1}{2^{n}}, \frac{1}{2^{n-1}}\right)}\right) V(x)+A, \frac{V(x)}{K\left(\frac{1}{2^{n}}, \frac{1}{2^{n-1}}\right)}+A^{\prime}\right\}\right]$.

Now, by the basic calculus on the proximal subgradients, we have

$$
\zeta \in \operatorname{co}\left\{\left(\frac{1}{K\left(\frac{1}{2^{n-1}}, \frac{1}{2^{n-2}}\right)}+\frac{1}{K\left(\frac{1}{2^{n+1}}, \frac{1}{2^{n}}\right)}\right) \partial_{P} V(x), \frac{1}{K\left(\frac{1}{2^{n}}, \frac{1}{2^{n-1}}\right)} \partial_{P} V(x) .\right\}
$$


Then, there exists $\zeta_{1}$ et $\zeta_{2}$ in $\partial_{P} V(x)$ and $t \in[0,1]$ such that

$$
\begin{aligned}
\zeta & =t\left(\frac{1}{K\left(\frac{1}{2^{n-1}}, \frac{1}{2^{n-2}}\right)}+\frac{1}{K\left(\frac{1}{2^{n}}, \frac{1}{2^{n-1}}\right)}\right) \zeta_{1}+(1-t) \frac{1}{K\left(\frac{1}{2^{n}}, \frac{1}{2^{n-1}}\right)} \zeta_{2} . \\
& =\left[t\left(\frac{1}{K\left(\frac{1}{2^{n-1}}, \frac{1}{2^{n-2}}\right)}+\frac{1}{K\left(\frac{1}{2^{n}}, \frac{1}{2^{n-1}}\right)}\right)+(1-t) \frac{1}{K\left(\frac{1}{2^{n}}, \frac{1}{2^{n-1}}\right)}\right] \hat{\zeta}
\end{aligned}
$$

where $\hat{\zeta} \in \partial_{P} V(x)$, because $\partial_{P} V(x)$ is a convex set. Now, we invoke the decrease property of $V, \inf _{u \in \mathcal{U}}\langle\hat{\zeta}, f(x, u)\rangle \leq-W(x)$. Then

$$
\inf _{u \in \mathcal{U}}\langle\zeta, f(x, u)\rangle \leq-\frac{1}{K\left(\frac{1}{2^{n}}, \frac{W(x)}{2^{n-1}}\right)},
$$

which gives the result.

Second case: If $n=1$, the proof is similar.

Now, note that for each $x \neq 0$, the sequence $\left(\mathcal{V}_{n}(x)\right)_{n \geq 0}$ is stationary, thus it converges. So, we can define

$$
\mathcal{V}(x):=\lim _{n \rightarrow \infty} \mathcal{V}_{n}(x)-C .
$$

where $C:=1-\sum_{n=0}^{+\infty} \frac{1}{2^{n+1} K\left(\frac{1}{2^{n+1}}, \frac{1}{2^{n}}\right)} \in[0,1]$ (because the Lipschitz constants have been chosen smaller than 1).

By the preceding lemma, $\mathcal{V}_{n}$ is always positive and for $x=0$,

$$
\mathcal{V}_{n}(0)=1-\sum_{k=1}^{n} \frac{1}{2^{k} K\left(\frac{1}{2^{k}}, \frac{1}{2^{k-1}}\right)} \rightarrow_{n \rightarrow \infty} 1-C=: \mathcal{V}(0) .
$$

We deduce that $\mathcal{V}(0)=0$ and then that $\mathcal{V}$ is positive definite. On the other hand, it is locally Lipschitz everywhere as a simple limit of Lipschitz functions (with the same constant in each compact set on $\mathbb{X}$ ) and it verifies the decreasing property (2.2) with a continuous positive definite function $\mathcal{W}$ defined as follows:

$$
\mathcal{W}(x):=\inf _{y \in \mathbb{X}}\{w(y)+\|x-y\|\}, \text { forall } x \in \mathbb{X}
$$

where

$$
w(x):= \begin{cases}W(x) & \text { if } x \notin S_{V}(1) \\ \frac{W(x)}{K\left(\frac{1}{2^{n+1}}, \frac{1}{2^{n}}\right)} & \text { if } x \in S_{V}\left(\frac{1}{2^{n}}\right) \backslash S_{V}\left(\frac{1}{2^{n+1}}\right) \\ 0 & \text { if } x=0 .\end{cases}
$$

The decrease property is an immediate consequence of (4.1).

To complete the proof of Theorem 2.7, we now have to prove the existence of a control-Lyapunov function which is continuous on $\mathbb{X}$ and locally Lipschitz outside the origin. We begin by defining a multifunction $F$, which is useful because uniformly bounded:

$$
\forall x \in \mathbb{X}, F(x):=\operatorname{cl} \quad \operatorname{co}\left\{\frac{f(x, u)}{1+\|f(x, u)\|} ; u \in \mathcal{U}\right\}
$$


We study the differential inclusion

$$
\dot{x}(t) \in F(x(t)) \quad \text { a.e. }
$$

This dynamic has the same properties as the system (2.1).

Proposition 4.2.

(i) $F$ is locally Lipschitz and compact convex valued;

(ii) The system $\dot{x}(t) \in F(x(t))$ is $G A C$.

Proof. (i) We omit the proof.

(ii) Let $x \in \mathbb{X}$ with $\|x\| \leq R$ be given. By assumption, there is a trajectory $x(\cdot)$ of $(2.1)$ on $[0, \infty)$ which verifies the assumptions of Global Asymptotic Controllability (Definition 2.3). We set

$$
\phi(t):=\int_{0}^{t}[1+\|\dot{x}(s)\|] d s
$$

and we define a function $\tilde{x}$ on $[0, \infty]$ by

$$
\tilde{x}(\tau):=x(t)
$$

where $t=t(\tau)$ is determined in $[0, \infty]$ by

$$
\tau=\int_{0}^{t}[1+\|\dot{x}(s)\|] d s
$$

(this change of variables or time scale is known as the Erdmann Transform.) Then

$$
\frac{d \tilde{x}}{d \tau}=\frac{\dot{x}(t)}{1+\|\dot{x}(t)\|} \in \tilde{\Gamma}(F x(\tau)) \quad \text { a.e. },
$$

so that $\tilde{x}$ is a $F$-trajectory.

But by construction, for all $\tau \geq 0,\|\tilde{x}(\tau)\| \leq M(R)$ and if $\tau \geq \phi(T(r, R))$ then $\|\tilde{x}(\tau)\| \leq r$.

The trajectory $x(\cdot)$ remains in the ball $M(R) \bar{B}$, so if $N_{R}$ denotes the maximum of $\|f(x, u)\|$ for $x \in M(R) \bar{B}$ and $u \in U$ (finite by the assumption 2.2), we have

$$
\forall t \geq 0, \phi(t) \leq t\left(1+N_{R}\right) .
$$

We deduce that if $\tau \geq T(r, R)\left(1+N_{R}\right)$, then $\tau \geq \phi(T(r, R)$ and consequently $\|\tilde{x}(\tau)\| \leq r$.

The new differential inclusion $\dot{x} \in F(x)$ is GAC with suitable constants $M(R)$ and $\tilde{T}(r, R):=T(r, R)\left(1+N_{R}\right)$.

We shall use the notation $M(\cdot)$ and $\tilde{T}(\cdot, \cdot)$ for the constant of global asymptotic stability of $F$.

REMARK 4.3. We have in fact by a similar proof the following property.

Proposition 4.4. Let $\beta: \mathbb{X} \longrightarrow \mathbb{R}_{>0}$ locally Lipschitz. Then the differential inclusion

$$
\dot{x}(t) \in \beta(x(t)) F(x(t)) \quad \text { a.e. }
$$

is locally Lipschitz with convex compact values and is GAC with appropriate constants $M(R) \downarrow 0$ and $\tilde{T}_{\beta}(r, R)=T(r, R) \max _{x \in M(R) \bar{B}}\left\{\beta(x)^{-1}\right\}$.

IGD, , , 


\section{First Step:}

We proceed to define a first multifunction $\Gamma_{0}$ as follows:

$$
\Gamma_{0}(x):= \begin{cases}{\left[1+(\|x\|-M(1)) \frac{\tilde{T}\left(\frac{1}{2}, 1\right)}{M(1)^{2}}\right]^{-1} F(x)} & \text { for }\|x\| \geq M(1) \\ F(x) & \text { for } 1 \leq\|x\| \leq M(1) \\ F(x)+4[1-\|x\|] \bar{B} & \text { for } \frac{1}{2} \leq\|x\| \leq 1 \\ F(x)+2 \bar{B} & \text { for }\|x\| \leq \frac{1}{2}\end{cases}
$$

By construction (and by the proposition 4.4), we have immediately the following lemma.

LEMMA 4.5. $\Gamma_{0}$ is compact convex valued, locally Lipschitz, uniformly bounded (by 1) and the differential inclusion $\dot{x} \in \Gamma_{0}(x(t))$ is GAC.

On the other hand, $\bar{B} \subset \Gamma_{0}(x)$ for all $x$ in $\frac{1}{2} \bar{B}$. Hence, the theorem 3.5 can be applied with $\mathcal{T}=\mathcal{T}_{0}:=\frac{1}{2} \bar{B}$ and $L=L_{0}:=1$. So we define the value function

$$
V_{0}(x):=\inf \left\{T: x(0)=x, \dot{x}(t) \in F(x(t)) \text { a.e. and } x(T) \in \frac{1}{2} \bar{B}\right\},
$$

for all $x \in \mathbb{X}$.

Lemma 4.6. $V_{0}$ est locally Lipschitz on $\mathbb{X}$, positive, proper and for all $x \notin B$,

$$
\forall \zeta \in \partial_{P} V_{0}(x), \min _{v \in F(x)}\langle\zeta, v\rangle \leq-1 .
$$

Proof. This is an easy corollary of the theorem 3.5 .

We set $m_{0}:=\max \left\{V_{0}(x) ;\|x\| \leq 1\right\}$ and $S_{0}:=\left\{x ; V_{0}(x) \leq m_{0}\right\}$.

We define a new function $\tilde{V}_{0}$ as follows:

$$
\tilde{V}_{0}(x):=\max \left\{0, V_{0}(x)-m_{0}\right\} .
$$

LEMMA 4.7.

(a) $\tilde{V}_{0}(x)=0 \Longleftrightarrow x \in S_{0}$;

(b) $\bar{B} \subset S_{0} \subset 3 M(1) \bar{B}$;

(c) $\forall x \notin S_{0}, \forall \zeta \in \partial_{P} \tilde{V}_{0}(x), \min _{v \in F(x)}\langle\zeta, v\rangle \leq-1$.

Proof. (a) Obvious by the definition of $\tilde{V}_{0}$.

(b) The first inclusion is given by the definition of $\tilde{V}_{0}$. However, the second one is less easy. Since the system $\dot{x} \in F(x))$ is GAC, for all $x \in \bar{B}$ there exists a $F$-trajectory $x(\cdot)$ such that

(1) $x(0)=\alpha$ et $\dot{x}(t) \in F(x(t))$ p.p,

(2) $\forall t \geq 0,\|x(t)\| \leq M(1)$,

(3) $\forall t \geq \tilde{T}\left(\frac{1}{2}, 1\right),\|x(t)\| \leq \frac{1}{2}$.

Now, since $\Gamma_{0}, \forall x \in M(1) \bar{B} \quad F(x) \subset \Gamma_{0}(x)$, then

$$
V_{0}(\alpha) \leq \tilde{T}\left(\frac{1}{2}, 1\right) .
$$

Consequently, $m_{0} \leq \tilde{T}\left(\frac{1}{2}, 1\right)$.

Let us consider now $\alpha \in \mathbb{X}$ such that $\|\alpha\| \geq 3 M(1)$. 
We remark that for $\|x\| \geq 2 M(1)$, we have

$$
\left\|\Gamma_{0}(x)\right\| \leq\left[1+\frac{\tilde{T}\left(\frac{1}{2}, 1\right)}{M(1)}\right]^{-1} .
$$

Then the time used for a $\Gamma_{0}$ trajectory with initial condition $\alpha$ to reach the ball $2 M(1) \bar{B}$ is greater than $\left[1+\frac{\tilde{T}\left(\frac{1}{2}, 1\right)}{M(1)}\right] M(1)$.

Hence, $V_{0}(\alpha) \geq M(1)+\tilde{T}\left(\frac{1}{2}, 1\right)>m_{0}$.

Consequently, $S_{0} \subset 3 M(1) \bar{B}$.

(c) This last assertion is a consequence of Lemma 4.6.

\section{Second step}

We now define a value function with a decrease property which holds closer to the origin. We set

$$
\Gamma_{1}(x):= \begin{cases}{\left[1+\left(\|x\|-M\left(\frac{1}{2}\right)\right) \frac{\tilde{T}\left(\frac{1}{4}, \frac{1}{2}\right)}{M\left(\frac{1}{2}\right)^{2}}\right]^{-1} F(x)} & \text { for }\|x\| \geq M\left(\frac{1}{2}\right) \\ F(x) & \text { for } \frac{1}{2} \leq\|x\| \leq M\left(\frac{1}{2}\right) \\ F(x)+8\left[\frac{1}{2}-\|x\|\right] \bar{B} & \text { for } \frac{1}{4} \leq\|x\| \leq \frac{1}{2} \\ F(x)+2 \bar{B} & \text { for }\|x\| \leq \frac{1}{4}\end{cases}
$$

We have immediatly the following result.

Lemma 4.8. $\Gamma_{1}$ is compact convex valued and the system $\dot{x}(t) \in \Gamma_{1}(x(t))$ is $G A C$ (with possible constants $M_{1}(R)=M(R) \downarrow 0$ and $\tilde{T}_{1}(r, R)$ ).

We need an auxiliary function with the local Lipschitz property.

We define for all $x \in \mathbb{X}$ :

$$
B_{0}(x):=\max \left\{V_{0}(y):\|y\| \leq\|x\|+M(1)\right\} .
$$

As before, the new multifunction leads to a value function $R_{1}$ associated to the set $\mathcal{A}_{1}:=\frac{1}{4} \bar{B}$. We set for all $x$ in $\mathbb{X}$ :

$$
R_{1}(x):=\inf \left\{\int_{0}^{T} L_{1}(x(t)) d t: x(0)=x, \dot{x} \in \Gamma_{1}(x) \text { a.e. and } x(T) \in \tau_{1}\right\}
$$

where $L_{1}(x):=1+\max \{0,\|x\|-3 M(1)\} \frac{B_{0}(x)}{\rho_{1} M(1)^{2}}$ and

$$
\rho_{1}:=\frac{m_{0} / 2}{m_{0}\left[1+\left(3 M(1)-M\left(\frac{1}{2}\right)\right) \frac{\tilde{T}\left(\frac{1}{4}, \frac{1}{2}\right)}{M\left(\frac{1}{2}\right)^{2}}\right]+\tilde{T}_{1}\left(\frac{1}{4}, 1\right)} \leq 1 .
$$

The theorem 3.5 gives the following lemma.

LEMMA 4.9 .

(a) $R_{1}$ is locally Lipschitz on $\mathbb{X}$;

(b) $\forall\|x\| \geq \frac{1}{2}, \forall \zeta \in \partial_{P} R_{1}(x), \min _{v \in F(x)}\langle\zeta, v\rangle \leq-L_{1}(x)$.

Proof. $L_{1}$ and $\Gamma_{1}$ being locally Lipschitz and the system associated to $\Gamma_{1}$ GAC, $R_{1}$ is finite everywhere and the theorem 3.5 proves the assertions.

As in the first step, we're going to evaluate the size of a certain level set given by $R_{1}$. We set $m_{R_{1}}:=\max \left\{R_{1}(y): y \in \frac{1}{2} \bar{B}\right\}$ and

$$
S_{R_{1}}\left(m_{R_{1}}\right)=\left\{x: R_{1}(x) \leq m_{R_{1}}\right\} .
$$


By the proposition 4.2 , for any $x \in \frac{1}{2} \bar{B}$, there exists a $F$-trajectory $x($.$) such$ that

(1) $x(0)=x$,

(2) $\forall t \geq 0,\|x(t)\| \leq M\left(\frac{1}{2}\right)$,

(3) $\forall t \geq \tilde{T}\left(\frac{1}{4}, \frac{1}{2}\right),\|x(t)\| \leq \frac{1}{4}$.

Moreover, $\forall x \in M\left(\frac{1}{2}\right) \bar{B} \quad F(x) \subset \Gamma_{1}(x)$, and $L_{1}(x)=1$, then

$$
R_{1}(x) \leq \tilde{T}\left(\frac{1}{4}, \frac{1}{2}\right)
$$

Consequently, $m_{R_{1}} \leq \tilde{T}\left(\frac{1}{4}, \frac{1}{2}\right)$.

Now, we consider an initial state $\alpha$ such that $\|\alpha\| \geq 3 M\left(\frac{1}{2}\right)$.

We remark that for $\|x\| \geq 2 M(1)$, we have

$$
\left\|\Gamma_{1}(x)\right\| \leq\left[1+\frac{\tilde{T}\left(\frac{1}{4}, \frac{1}{2}\right)}{M\left(\frac{1}{2}\right)}\right]^{-1} .
$$

Then the time used for a $\Gamma_{1}$ trajectory with initial condition $\alpha$ to reach the ball $2 M\left(\frac{1}{2}\right) \bar{B}$ is greater than $\left[1+\frac{\tilde{T}\left(\frac{1}{4}, \frac{1}{2}\right)}{M\left(\frac{1}{2}\right)}\right] M\left(\frac{1}{2}\right)$.

Hence, $L_{1} \geq 1$ implies $R_{1}(\alpha) \geq M\left(\frac{1}{2}\right)+\tilde{T}\left(\frac{1}{4}, \frac{1}{2}\right)>m_{R_{1}}$.

Consequently:

$$
S_{R_{1}}\left(m_{R_{1}}\right) \subset 3 M\left(\frac{1}{2}\right) \bar{B} .
$$

Indeed, The proof gives the following lemma.

LEMMA 4.10. $\frac{1}{2} \bar{B} \subset S_{R_{1}}\left(m_{R_{1}}\right) \subset 3 M\left(\frac{1}{2}\right) \bar{B}$.

We want now to compare $R_{1}$ with $V_{0}$.

LEMMA 4.11.

(a) $\forall x \in S_{0}, \rho_{1} R_{1}(x) \leq \frac{m_{0}}{2}$;

(b) If $\|x\| \geq 5 M(1)$, then $V_{0}(x) \leq \rho_{1} R_{1}(x)$.

Proof. (a) Let $x \in S_{0}$. Indeed, there exists a $\Gamma_{0}$-trajectory $x(\cdot)$ which relies $x$ to the set $\bar{B}$ with time $T_{x} \leq V_{0}(x) \leq m_{0}$. Hence, $\forall t \geq 0, x(t) \in S_{0} \subset$ $3 M(1) \bar{B}$ (by Lemma $4.7(\mathrm{~b})$ ). In the zone $\|x\| \in[1,3 M(1)]$ we can write $\Gamma_{1}(x) \subset \beta(x) \Gamma_{0}(x)$ with $\beta(x)$ as follows (assuming that $M\left(\frac{1}{2} \geq 1\right)$ :

$$
\beta(x):= \begin{cases}1 & \text { if }\|x\| \in\left[\frac{1}{2}, M\left(\frac{1}{2}\right)\right] \\ {\left[1+\left(\|x\|-M\left(\frac{1}{2}\right)\right) \frac{\tilde{T}\left(\frac{1}{4}, \frac{1}{2}\right)}{M\left(\frac{1}{2}\right)^{2}}\right]^{-1}} & \text { if }\|x\| \in\left[M\left(\frac{1}{2}\right), M(1)\right] \\ \frac{\left[1+\left(\|x\|-M\left(\frac{1}{2}\right)\right) \frac{\tilde{T}\left(\frac{1}{4}, \frac{1}{2}\right)}{M\left(\frac{1}{2}\right)^{2}}\right]^{-1}}{\left[1+(\|x\|-M(1)) \frac{\tilde{T}\left(\frac{1}{2}, 1\right)}{M(1)^{2}}\right]^{-1}} & \text { if }\|x\| \in[M(1), 3 M(1)]\end{cases}
$$

We observe that if $M\left(\frac{1}{2}\right)<1$, we have to omit it in the definition of $\beta$. Now, an appropriate change of variables (see Proposition 4.4) provides that there exists a $\Gamma_{1}$-trajectory $x(\cdot)$ which remains in $3 M(1) \bar{B}$ and drives $x$ to $\bar{B}$ with a time $T \leq T_{x} \max _{\|x\| \in[1,3 M(1)]} \beta(x)^{-1}$.

Thus, we calculate $T \leq m_{0}\left[1+\left(3 M(1)-M\left(\frac{1}{2}\right)\right) \frac{\tilde{T}\left(\frac{1}{4}, \frac{1}{2}\right)}{M\left(\frac{1}{2}\right)^{2}}\right]$.

Now, we can extend this trajectory until $\mathcal{T}_{1}$ with the following property (by Lemma 4.8): $\forall t \geq T, x(t) \in M(1) \bar{B}$ and $x\left(T+\tilde{T}_{1}\left(\frac{1}{4}, 1\right)\right) \in \frac{1}{4} \bar{B}$. In 
so doing, we have constructed a trajectory which remains in $3 M(1) \bar{B}$ ( where $L_{1}=1$ ) and reaches the set $\mathcal{T}_{1}$.

Consequently, $R_{1}(x) \leq m_{0}\left[1+\left(3 M(1)-M\left(\frac{1}{2}\right)\right) \frac{\tilde{T}\left(\frac{1}{4}, \frac{1}{2}\right)}{M\left(\frac{1}{2}\right)^{2}}\right]+\tilde{T}_{1}\left(\frac{1}{4}, 1\right)$.

We conclude by the definition of $\rho_{1}$.

(b) Let $x$ such that $\|x\| \geq 5 M(1)$. By the definition of $B_{0}$ we have

$\|y\| \geq\|x\|-M(1) \Longrightarrow B_{0}(y) \geq V_{0}(x) \Longrightarrow L_{1}(y) \geq 1+\frac{V_{0}(x)}{\rho_{1} M(1)^{2}}$.

On the other hand, the time required for driving from $\{\|y\| \geq\|x\|-$ $M(1)\}$ to $\{\|y\| \geq\|x\|-2 M(1)\}$ is greater than $M(1)$ (the dynamic is bounded by 1 ). Consequently,

$$
\begin{aligned}
R_{1}(x) & \geq M(1)\left[1+\frac{V_{0}(x)}{\rho_{1} M(1)}\right] \\
& \geq \frac{V_{0}(x)}{\rho_{1}} .
\end{aligned}
$$

We finish this step by defining a new function $V_{1}$ as follows.

$$
\forall x \in \mathbb{X}, V_{1}(x):=\min \left\{\tilde{V}_{0}(x)+m_{0}, \rho_{1} R_{1}(x)\right\} .
$$

We set $m_{1}:=\max \left\{V_{1}(x): x \in \frac{1}{2} \bar{B}\right\}$ and $S_{1}:=\left\{x: V_{1}(x) \leq m_{1}\right\}$. We have the following lemma.

Lemma 4.12. $V_{1}$ is locally Lipschitz on $\mathbb{X}$. Moreover, we have,

(a) $m_{1} \leq \frac{m_{0}}{2}$;

(b) $\forall x \in S_{0} \cup S_{1}, V_{1}(x)=\rho_{1} R_{1}(x)$;

(c) $\frac{1}{2} \bar{B} \subset S_{1} \subset 3 M\left(\frac{1}{2}\right) \bar{B}$;

(d) If $\|x\| \geq 5 M(1)$ then $V_{1}(x)=V_{0}(x)$.

(e) For $\frac{1}{2} \leq\|x\| \leq 5 M(1), \forall \zeta \in \partial_{P} V_{1}(x), \min _{v \in F(x)}\langle\zeta, v\rangle \leq-\rho_{1}$;

(f) For $\|x\|>5 M(1), \forall \zeta \in \partial_{P} V_{1}(x), \min _{v \in F(x)}\langle\zeta, v\rangle \leq-1$.

Proof. (a) By the Lemma 4.11 (a), for any $x \in S_{0}, \rho_{1} R_{1}(x) \leq \frac{m_{0}}{2}$. Hence by definition of $V_{1}, \forall x \in S_{0}, V_{1}(x)=\rho_{1} R_{1}(x)$. Thus we conclude by remarking that $\frac{1}{2} \bar{B} \subset S_{0}$. We have in fact $m_{1}=\rho_{1} m_{R_{1}}$.

(b) let $x \in S_{0} \cup S_{1}$. If $x \in S_{0}$, we have shown the equality in the first assertion. Otherwise, $V_{1}(x) \leq m_{1} \leq \frac{m_{0}}{2}$ implies the equality.

(c) If $x \in S_{1}$ then $V_{1}(x)=\rho_{1} R_{1}(x) \leq m_{1}$. And by the remark of (a), $R_{1}(x) \leq m_{R_{1}}$ which gives the inclusion.

(d) For $\|x\| \geq 5 M(1)$, we have that $V_{0}(x)=\tilde{V}_{0}(x)+m_{0}$ (because $S_{0} \subset$ $3 M(1) \bar{B})$. We conclude by Lemma $4.11(\mathrm{~b})$.

(e) Let $x \in \mathbb{R}^{N}$ such that $\frac{1}{2} \leq\|x\| \leq 5 M(1)$ and $\zeta \in \partial_{P} V_{1}(x)$. We recall the definition of $V_{1}(x)$.

$$
V_{1}(x):=\min \left\{\tilde{V}_{0}(x)+m_{0}, \rho_{1} R_{1}(x)\right\} .
$$

First Case: The minimum is attained for the second term.

Then $\zeta \in \partial_{P} \rho_{1} R_{1}(x)=\rho_{1} \partial_{P} R_{1}(x)$

We conclude by Lemma 4.9 (b).

Second Case: the minimum is attained for the first term and not for the IGD, , , 
second one. In this case, $x \notin S_{0}$ and $\zeta \in \partial_{P}\left(\tilde{V}_{0}+m_{0}\right)(x)=\partial_{P} \tilde{V}_{0}(x)$. We conclude by Lemma 4.7 (c).

(f) This is an easy consequence of the Lemma 4.11(b).

\section{Third Step}

We finish the construction of the sequence $\left(V_{n}\right)_{n \geq 0}$ by induction on $n$. Assume $\left(V_{k}, \mathcal{A}_{k}, L_{k}, R_{k}, \Gamma_{k}\right)$ have been already defined for $1 \leq k \leq n$ with the following properties.

1) $\frac{1}{2^{k}} \bar{B} \subset S_{k} \subset 3 M\left(\frac{1}{2^{k}}\right) \bar{B}$

2) For $\|x\| \geq 5 M\left(\frac{1}{2^{k-1}}\right), V_{k}(x)=V_{k-1}(x)$;

3) For $\frac{1}{2^{k}} \leq\|x\| \leq 5 M\left(\frac{1}{2^{k-1}}\right), \forall \zeta \in \partial_{P} V_{k}(x), \min _{v \in F(x)}\langle\zeta, v\rangle \leq-\rho_{k}$;

4) $L_{k}=1$ on the ball $3 M\left(\frac{1}{2^{k-1}}\right) \bar{B}$;

5) $\forall x \in \mathbb{R}^{N}, V_{k}(x)=0 \Longleftrightarrow x \in \frac{1}{2^{k+1}} \bar{B}$;

6) $\forall k \in[1, n], m_{k} \leq \frac{m_{k-1}}{2}$, and $\rho_{k} \leq \rho_{k-1} \leq 1=$ : $\rho_{0}$.

With the following definitions:

$$
m_{k}:=\max \left\{V_{k}(x) ;\|x\| \leq \frac{1}{2^{k}}\right\}, \text { and } S_{k}:=\left\{x ; V_{k}(x) \leq m_{k}\right\},
$$

where $\rho_{k}$ are some positive constants.

As before, we can define a new function $V_{n+1}$. We proceed as follows; for all $x \in \mathbb{X}$, we set $\Gamma_{n+1}(x):=$

$$
\begin{cases}{\left[1+\left(\|x\|-M\left(\frac{1}{2^{n+1}}\right)\right) \frac{\tilde{T}\left(\frac{1}{2^{n+2}}, \frac{1}{2^{n+1}}\right)}{M\left(\frac{1}{2^{n+1}}\right)^{2}}\right]^{-1} F(x)} & \text { if }\|x\| \geq M\left(\frac{1}{2^{n+1}}\right) \\ F(x) & \text { if }\|x\| \in\left[\frac{1}{2^{n+1}}, M\left(\frac{1}{2^{n+1}}\right)\right] \\ F(x)+2^{n+3}\left[\frac{1}{2^{n+1}}-\|x\|\right] \bar{B} & \text { if }\|x\| \in\left[\frac{1}{2^{n+2}}, \frac{1}{2^{n+1}}\right] \\ F(x)+2 \bar{B} & \text { if }\|x\| \leq \frac{1}{2^{n+2}}\end{cases}
$$

We need as before an auxiliary function with the local Lipschitz property. We define for all $x \in \mathbb{X}$ :

$$
B_{n}(x):=\max \left\{V_{n}(y):\|y\| \leq\|x\|+M\left(\frac{1}{2^{n}}\right)\right\} .
$$

From this multifunction, we define a value function associated to the set $\mathcal{T}_{n+1}:=\frac{1}{2^{n+2}} \bar{B}$. We set for any $x \in \mathbb{X}$,

$R_{n+1}(x):=\inf \left\{\int_{0}^{T} L_{n+1}(x(t)) d t: x(0)=x, \dot{x} \in \Gamma_{n+1}(x)\right.$ and $\left.x(T) \in \mathcal{T}_{n+1}\right\}$

where $L_{n+1}(x):=1+\max \left\{0,\|x\|-3 M\left(\frac{1}{2^{n}}\right)\right\} \frac{B_{n}(x)}{\rho_{n+1} M\left(\frac{1}{2^{n}}\right)^{2}}$ with

$$
\rho_{n+1}:=\frac{\rho_{n} m_{n} / 2}{m_{n}\left[1+\left(3 M\left(\frac{1}{2^{n}}\right)-M\left(\frac{1}{2^{n+1}}\right)\right) \frac{\tilde{T}\left(\frac{1}{2^{n+2}}, \frac{1}{2^{n+1}}\right)}{M\left(\frac{1}{2^{n+1}}\right)^{2}}\right]+\tilde{T}_{n+1}\left(\frac{1}{2^{n+2}}, \frac{1}{2^{n}}\right)} \leq \rho_{n} .
$$

The differential inclusion $\left.\dot{x} \in \Gamma_{n+1}(x)\right)$ is GAC, we denote by $\tilde{T}_{n+1}(\cdot, \cdot)$ its new constant (we saw that we can choose $\tilde{M}_{n+1}=M$ ). On the other hand, we set

$$
\begin{gathered}
m_{R_{n+1}}:=\max \left\{R_{n+1}(y): y \in \frac{1}{2^{n+1}} \bar{B}\right\}, \\
\text { and } S_{R_{n+1}}\left(m_{R_{n+1}}\right):=\left\{x: R_{n+1}(x) \leq m_{R_{n+1}}\right\} .
\end{gathered}
$$


LEMMA 4.13.

(a) $R_{n+1}$ is locally Lipschitz on $\mathbb{X}$;

(b) $\forall\|x\| \geq \frac{1}{2^{n+1}}, \forall \zeta \in \partial_{P} R_{n+1}(x), \min _{v \in F(x)}\langle\zeta, v\rangle \leq-L_{n+1}(x)$;

(c) $\frac{1}{2^{n+1}} \bar{B} \subset S_{R_{n+1}}\left(m_{R_{n+1}}\right) \subset 3 M\left(\frac{1}{2^{n+1}}\right) \bar{B}$;

(d) $\forall x \in S_{n}, \rho_{n+1} R_{n+1}(x) \leq \frac{m_{n}}{2}$;

(e) If $\|x\| \geq 5 M\left(\frac{1}{2^{n}}\right)$, then $V_{n}(x) \leq \rho_{n+1} R_{n+1}(x)$.

Proof. (a) and (b) are evident by the theorem 2.7. The assertion (c) is proved as before (Lemma 4.10). We prove now (d) and (e), we begin with (e).

Let there be given $\|x\| \geq 5 M\left(\frac{1}{2^{n}}\right)$. By the definition of $B_{n}$ we have

$$
\|y\| \geq\|x\|-M\left(\frac{1}{2^{n}}\right) \Longrightarrow B_{n}(y) \geq V_{n}(x) \Longrightarrow L_{n+1}(y) \geq 1+\frac{V_{n}(x)}{\rho_{n+1} M\left(\frac{1}{2^{n}}\right)^{2}} .
$$

On the other hand, the time required for driving from $\left\{\|y\| \geq\|x\|-M\left(\frac{1}{2^{n}}\right)\right\}$ to $\left\{\|y\| \geq\|x\|-2 M\left(\frac{1}{2^{n}}\right)\right\}$ is greater than $M\left(\frac{1}{2^{n}}\right)$ (the dynamic is bounded by 1$)$. Consequently,

$$
\begin{aligned}
R_{n+1}(x) & \geq M\left(\frac{1}{2^{n}}\right)\left[1+\frac{V_{n}(x)}{\rho_{n+1} M\left(\frac{1}{2^{n}}\right)}\right] \\
& \geq \frac{V_{n}(x)}{\rho_{n+1}} .
\end{aligned}
$$

We prove now (d). Let $x \in S_{n}$. Indeed, there exists a $\Gamma_{n}$-trajectory $x(\cdot)$ which relies $x$ to the set $\frac{1}{2^{n}} \bar{B}$ with time $T_{x} \leq V_{n}(x) \leq m_{n}$ and which remains in $S_{n}$ (because $S_{n} \subset 3 M\left(\frac{1}{2^{n}} \bar{B}\right.$ and $L_{n}=1$ on $3 M\left(\frac{1}{2^{n}} \bar{B}\right)$. In the zone $\|x\| \in\left[\frac{1}{2^{n}}, 3 M\left(\frac{1}{2^{n}}\right)\right]$ we can write $\Gamma_{n+1}(x) \subset \beta(x) \Gamma_{n}(x)$ with $\beta(x)$ as follows (assuming that $M\left(\frac{1}{2^{n+1}}\right) \geq \frac{1}{2^{n}}$; we adapt otherwise): $\beta(x):=$

$$
\begin{cases}1 & \text { for }\|x\| \in\left[\frac{1}{2^{n}}, M\left(\frac{1}{2^{n+1}}\right)\right] \\ {\left[1+\left(\|x\|-M\left(\frac{1}{2^{n+1}}\right)\right) \frac{\tilde{T}\left(\frac{1}{2^{n+2}}, \frac{1}{2^{n+1}}\right)}{2^{n+1}}\right)^{2}} & \text { for }\|x\| \in\left[M\left(\frac{1}{2^{n+1}}\right), M\left(\frac{1}{2^{n}}\right)\right] \\ \frac{\left[1+\left(\|x\|-M\left(\frac{1}{2^{n+1}}\right)\right) \frac{\tilde{T}\left(\frac{1}{2^{n+2}}, \frac{1}{2^{n+1}}\right)}{M\left(\frac{1}{2^{n+1}}\right)^{2}}\right]^{-1}}{\left[1+\left(\|x\|-M\left(\frac{1}{2^{n}}\right)\right) \frac{\tilde{T}\left(\frac{1}{2^{n+1}}, 2^{n}\right)}{M\left(\frac{1}{2^{n}}\right)^{2}}\right]^{-1}} & \text { for }\|x\| \in\left[M\left(\frac{1}{2^{n}}\right), 3 M\left(\frac{1}{2^{n}}\right)\right]\end{cases}
$$

An appropriate change of variables (see Proposition 4.4) provides that there exists a $\Gamma_{n+1}$-trajectory $x(\cdot)$ which remains in $3 M\left(\frac{1}{2^{n}}\right) \bar{B}$ and drives $x$ to $\frac{1}{2^{n}} \bar{B}$ with a time $T \leq T_{x} \max _{\|x\| \in\left[\frac{1}{2^{n}}, 3 M\left(\frac{1}{2^{n}}\right)\right]} \beta(x)^{-1}$.

Thus, we calculate $T \leq m_{n}\left[1+\left(3 M\left(\frac{1}{2^{n}}\right)-M\left(\frac{1}{2^{n+1}}\right)\right) \frac{\tilde{T}\left(\frac{1}{2^{n+2}}, \frac{1}{2^{n+1}}\right)}{M\left(\frac{1}{2^{n+1}}\right)^{2}}\right]$.

Now, we can extend this trajectory until $\mathcal{T}_{n+1}$ with the following property (by Lemma 4.8): $\forall t \geq T, x(t) \in M\left(\frac{1}{2^{n}}\right) \bar{B}$ and $x\left(T+\tilde{T}_{n+1}\left(\frac{1}{2^{n+2}}, \frac{1}{2^{n}}\right)\right) \in \mathcal{T}_{n+1}$. In this way, we have constructed a trajectory which remains in $3 M\left(\frac{1}{2^{n}}\right) \bar{B}$ ( where $L_{n+1}=1$ ) and reaches the set $\mathcal{T}_{n+1}$.

Consequently,

$R_{n+1}(x) \leq m_{n}\left[1+\left(3 M\left(\frac{1}{2^{n}}\right)-M\left(\frac{1}{2^{n+1}}\right)\right) \frac{\tilde{T}\left(\frac{1}{2^{n+2}}, \frac{1}{2^{n+1}}\right)}{M\left(\frac{1}{2^{n+1}}\right)^{2}}\right]+\tilde{T}_{1}\left(\frac{1}{2^{n+2}}, \frac{1}{2^{n}}\right)$.

We conclude by the definition of $\rho_{n+1}$. IGD, , , 
We can now define the new function $V_{n+1}$. We set for all $x \in \mathbb{X}$,

$$
V_{n+1}(x):=\min \left\{\tilde{V}_{n}(x)+m_{n}, \rho_{n+1} R_{n+1}(x)\right\},
$$

where $\tilde{V}_{n}(x):=\max \left\{0, V_{n}(x)-m_{n}\right\}$.

As before, we consider

$$
m_{n+1}:=\max \left\{V_{n+1}(x): x \in \frac{1}{2^{n+1}} \bar{B}\right\} \text { and } S_{n+1}:=\left\{x: V_{n+1}(x) \leq m_{n+1}\right\} .
$$

We have the following lemma.

Lemma 4.14. $V_{n+1}$ is locally Lipschitz on $\mathbb{X}$. Moreover, we have

(a) $m_{n+1} \leq \frac{m_{n}}{2}$;

(b) $\frac{1}{2^{n+1}} \bar{B} \subset S_{n+1} \subset 3 M\left(\frac{1}{2^{n+1}}\right) \bar{B}$;

(c) If $\|x\| \geq 5 M\left(\frac{1}{2^{n}}\right)$, then $V_{n+1}(x)=V_{n}(x)$;

(d) For $\frac{1}{2^{n+1}} \leq\|x\| \leq 5 M\left(\frac{1}{2^{n}}\right), \forall \zeta \in \partial_{P} V_{n+1}(x), \min _{v \in F(x)}\langle\zeta, v\rangle \leq-\rho_{n+1}$.

Proof. The proof is similar to the proof of Lemma 4.12. This is let to the reader.

\section{Fourth Step: The Function $V$}

We study the convergence of the sequence $\left(V_{n}\right)_{n \geq 0}$. For that, we need a last lemma.

Lemma 4.15. $\forall 0 \leq k \leq n, \forall x \in S_{k}, V_{n}(x) \leq m_{k}$.

Proof. We do an inductive proof. The result being already proven for $\mathrm{n}=1$ (Lemma 4.12), assume that we have proved the result for $n \geq 1$; we establish the property for $n+1$.

Let us consider $0 \leq k \leq n+1$ and $x \in S_{k}$.

First Case: $k \leq n$.

If $x \notin S_{n}$, then by definition of $V_{n}, V_{n}(x)=\tilde{V}_{n}(x)+m_{n}$. Hence, $V_{n+1}(x) \leq$ $V_{n}(x) \leq m_{k}$ by induction.

Otherwise, $x \in S_{n}$. In this case, $\tilde{V}_{n}(x)=0$ implies $V_{n+1}(x) \leq m_{n} \leq m_{k}$ by the property on the sequence $\left(m_{k}\right)$.

Second Case: $k=n+1$.

The property follows from the definition of $S_{n+1}$.

We can now conclude. Let us consider $K$ a compact set of $\mathbb{X} \backslash\{0\}$. Then, as $\lim _{n \rightarrow \infty} M\left(\frac{1}{2^{n}}\right)=0$, there exists a positive integer $n_{K}$ such that

$$
\|x\| \geq 5 M\left(\frac{1}{2^{n_{K}}}\right), \quad \forall x \in K .
$$

By the second property of the sequence $\left(V_{n}\right)_{n \geq 0}$, for any $n \geq n_{K}, V_{n}(x)=$ $V_{n_{K}}(x)$.

Hence, the sequence $\left(V_{n}(x)\right)_{n \geq 0}$ converges for all $x$ in $K$ and the limit is locally Lipschitz function in $\bar{K}$ (as a stationnar limit of locally Lipschitz functions). On the other hand, for any $n \geq 0, V_{n}(0)=0$; so we can define for all $x \in \mathbb{X}$,

$$
V(x):=\lim _{n \rightarrow \infty} V_{n}(x) .
$$

By the proof above, $V$ is locally Lipschitz on $\mathbb{X} \backslash\{0\}$, positive definite and proper (because $V_{n}=V_{0} \quad \forall n$ if $\|x\| \geq 5 M(1)$ ); we have to show that $V$ is continuous at the origin. This fact is a consequence of the preceding lemma. Let us consider $x_{p} \longrightarrow p \rightarrow \infty$. We want to show that $f\left(x_{p}\right) \longrightarrow p \rightarrow \infty 0$. 
Let $\epsilon>0$. There exists $n_{0} \geq 0$ such that $m_{n_{0}} \leq \epsilon$ (because $\left.m_{n} \leq \frac{m_{0}}{2^{n}}\right)$. Thus, by the last lemma, $\forall n \geq n_{0}, \forall x \in S_{n_{0}}, V_{n}(x) \leq \epsilon$.

But for $p$ sufficiently great $(p \geq P), x_{p} \in \frac{1}{2^{n_{0}}} \bar{B} \subset S_{n_{0}}$.

We deduce that $\forall n \geq n_{0}, \forall p \geq P, V_{n}\left(x_{p}\right) \leq \epsilon$. By passing to the limit: $\forall p \geq P, V\left(x_{p}\right) \leq \epsilon$, which gives the continuity on the origin.

Now, we set $\forall x \in \mathbb{X}$,

$$
w(x):= \begin{cases}\rho_{n} & \text { if } 5 M\left(\frac{1}{2^{n+1}}\right)<\|x\| \leq 5 M\left(\frac{1}{2^{n}}\right) \\ 1 & \text { if }\|x\|>5 M(1) \\ 0 & \text { if } x=0 .\end{cases}
$$

We can now define the function $W$ by

$$
\forall x \in \mathbb{X}, W(x):=\inf _{y \in \mathbb{X}}\{w(y)+\|x-y\|\} .
$$

$W$ is a positive definite and locally Lipschitz function. The decrease condition (2.2) is the consequence of the stationarity of the sequence $\left(V_{n}(x)\right)_{n \geq 0}$ outside the origin and of the Lemma 4.14 (d). This completes the proof of Theorem 2.7.

\section{Existence of A SEMiconcave CLF}

We begin by some preliminaries on the semiconcavity. It is easy to show that any semiconcave function in $\Omega$ is locally Lipschitz. Concave functions are of course, semiconcave. Another class of semiconcave functions is that of $C^{1}$ functions with locally Lipschitz gradient. Moreover we have the two following lemmas.

LEMMA 5.1. Let $\Psi: \mathbb{R} \longrightarrow \mathbb{R}$ be an increasing semiconcave function and $u: \Omega \longrightarrow \mathbb{R}$ be a semiconcave function on $\Omega$. Then $\Psi \circ u$ is a semiconcave function on $\Omega$.

Lemma 5.2. Let $u, v: \Omega \longrightarrow \mathbb{R}$ be two semiconcave functions on $\Omega$, then the function $\min \{u, v\}$ is semiconcave on $\Omega$.

A convenient way to build semiconcave approximations of a given function is provided by the method of inf-convolution, a standard tool in convex and non-smooth analysis. Let $\Omega$ be a subset of $\mathbb{X}$ and $u$ a positive function in $\Omega$. Define, for any $\alpha>0$,

$$
u_{\alpha}(x):=\inf _{y \in \Omega}\left\{u(y)+\alpha\|x-y\|^{2}\right\} .
$$

Lemma 5.3. Let $u: \mathbb{X} \longrightarrow \mathbb{R}$ be a locally Lipschitz and proper function. Then $u_{\alpha}$ is semiconcave on $\mathbb{X}$ (the infimum is attained in the definition of $u_{\alpha}$ ) and moreover, $u_{\alpha} \nearrow u$, as $\alpha \rightarrow+\infty$, locally uniformly in $\mathbb{X}$.

Proof. We leave the proof to the reader.

We can link the proximal subdifferentials of $u$ and its inf-convolution. We have the following Lemma. (We refer to [14, Theorem.5.1,p.44] for the proof.)

Lemma 5.4. Suppose that $x \in \mathbb{X}$ is such that $\partial_{P} u_{\alpha}(x)$ is nonempty. Then there exists a point $\bar{y} \in \mathbb{X}$ satisfying the following:

a) The infimum in (5.1) is attained uniquely at $\bar{y}$.

b) The proximal subgradient $\partial_{P} u_{\alpha}(x)$ is the singleton $\{2 \alpha(x-\bar{y})\}$. IGD, , 
c) $2 \alpha(x-\bar{y}) \in \partial_{P} u(\bar{y})$.

\section{Proof of Theorem 2.10}

By Theorem 2.7, there exists a control-Lyapunov pair for the system (2.1); without loss of generality, we can suppose that the function $W$ is 1-Lipschitz on $\mathbb{X}$ (othewise, we can set $\tilde{W}(x):=\inf _{y \in \mathbb{X}}\{W(y)+\|x-y\|\}$ ).

For any $0<r<R$, we define the following sets:

$$
S_{V}[r, R]:=\{x \in \mathbb{X}: V(x) \in[r, R]\} \text { and } S_{V}(R):=\{x \in \mathbb{X}: V(x) \leq R\} .
$$

Let there be given an integer $n \in \mathbb{N}^{*}$.

By the Lipschitz property of $f$ and $V$, we can consider $L_{f}^{n} \geq 1$ (respectively $L_{V}^{n} \geq 1$ ) the Lipschitz constant of $f(\cdot, u)$ (respectively of $V$ ) on the level set $S_{V}\left(M_{n}\right)$ where the constant $M_{n}$ is defined by

$$
M_{n}:=\max \left\{V(x): x \in S_{V}(11 n)+\bar{B}\right\} .
$$

On the other hand, we note $w_{n}$ the minimum of $W$ on $S_{V}\left[\frac{1}{2 n}, 11 n\right]$, and we set

$$
\alpha_{n}:=\max \left\{8 n\left(L_{V}^{n}\right)^{2}+1, \frac{2 L_{V}^{n}\left(1+L_{V}^{n} L_{f}^{n}\right)}{w_{n}}+1,11 n\right\} .
$$

We define by inf-convolution the function $V_{\alpha_{n}}$ as follows:

$$
V_{\alpha_{n}}(x):=\inf _{y \in \mathbb{X}}\left\{V(y)+\alpha_{n}\|x-y\|^{2}\right\} .
$$

Lemma 5.5. Let $x_{0} \in S_{V}\left(M_{n}\right)$. If the infimum in the definition of $V_{\alpha_{n}}\left(x_{0}\right)$ is attained at $\bar{y}$, then $\left\|x_{0}-\bar{y}\right\| \leq \min \left\{\frac{1}{8 n L_{V}^{n}}, \frac{w_{n}}{2\left(1+L_{V}^{n} L_{f}^{n}\right)}\right\}$ and

$$
V\left(x_{0}\right)-\frac{1}{8 n} \leq V_{\alpha_{n}}\left(x_{0}\right) \leq V\left(x_{0}\right) .
$$

Proof. If the infimum is attained for $\bar{y}$, then $V(\bar{y}) \leq V\left(x_{0}\right) \leq M_{n} \Longrightarrow \bar{y} \in$ $S_{V}\left(M_{n}\right)$. Hence, if $\left\|x_{0}-\bar{y}\right\|>\min \left\{\frac{1}{8 n L_{V}^{n}}, \frac{w_{n}}{2\left(1+L_{V}^{n} L_{f}^{n}\right)}\right\}$ then, by definition of $L_{f}^{n}$ and $L_{V}^{n}$ :

$$
\begin{aligned}
V_{\alpha_{n}}\left(x_{0}\right) & =V(\bar{y})+\alpha_{n}\left\|x_{0}-\bar{y}\right\|^{2} \\
& \geq V\left(x_{0}\right)-L_{V}^{n}\left\|x_{0}-\bar{y}\right\|+\alpha_{n}\left\|x_{0}-\bar{y}\right\|^{2} \\
& \geq V\left(x_{0}\right)+\left\|x_{0}-\bar{y}\right\|\left[\alpha_{n}\left\|x_{0}-\bar{y}\right\|-L_{V}^{n}\right] \\
& \geq V\left(x_{0}\right)+\left\|x_{0}-\bar{y}\right\|\left[\alpha_{n} \min \left\{\frac{1}{8 n L_{V}^{n}}, \frac{w_{n}}{2\left(1+L_{V}^{n} L_{f}^{n}\right)}\right\}-L_{V}^{n}\right] \\
& >V(x),
\end{aligned}
$$

we find a contradiction. Hence, $\left\|x_{0}-\bar{y}\right\| \leq \min \left\{\frac{1}{8 n L_{V}^{n}}, \frac{w_{n}}{2\left(1+L_{V}^{n} L_{f}^{n}\right)}\right\}$. On the other hand, we have found the estimate

$$
V_{\alpha_{n}}\left(x_{0}\right) \geq V\left(x_{0}\right)+\left\|x_{0}-\bar{y}\right\|\left[\alpha_{n}\left\|x_{0}-\bar{y}\right\|-L_{V}^{n}\right] .
$$

Consequently, $V_{\alpha_{n}}\left(x_{0}\right) \geq V\left(x_{0}\right)-L_{V}^{n}\left\|x_{0}-\bar{y}\right\|$ which implies the desired inequality by the bound on $\left\|x_{0}-\bar{y}\right\|$. 
Lemma 5.6. Let $x_{0} \in S_{V}\left[\frac{1}{2 n}, 11 n\right]$ and $\zeta \in \partial_{P} V_{\alpha_{n}}\left(x_{0}\right)$, then

$$
\inf _{u \in U}\left\langle\zeta, f\left(x_{0}, u\right)\right\rangle \leq-\frac{W\left(x_{0}\right)}{2} .
$$

Proof. By the Lemmas 5.4 and 5.5, the infimum in the definition of $V_{\alpha_{n}}\left(x_{0}\right)$ is attained uniquely at a point $\bar{y} \in S_{V}(11 n)$ which satisfies $\left\|x_{0}-\bar{y}\right\| \leq$ $\frac{w_{n}}{2\left(1+L_{V}^{n} L_{f}^{n}\right)}$ and such that $\zeta \in \partial_{P} V(\bar{y})$. Thus, by the Lipschitz properties of $f, V$ and $W$, we can write:

$$
\begin{aligned}
\inf _{u \in U}\left\langle\zeta, f\left(x_{0}, u\right)\right\rangle & \leq \inf _{u \in U}\langle\zeta, f(\bar{y}, u)\rangle+\sup _{u \in U}\|\zeta\|\left\|f\left(x_{0}, u\right)-f(\bar{y}, u)\right\| \\
& \leq-W(\bar{y})+L_{V}^{n} L_{f}^{n}\left\|x_{0}-\bar{y}\right\| \quad \text { (decrease condition) } \\
& \leq-W\left(x_{0}\right)+\left(1+L_{V}^{n} L_{f}^{n}\right)\left\|x_{0}-\bar{y}\right\| \\
& \leq-W\left(x_{0}\right)+\frac{w_{n}}{2} \leq-\frac{W\left(x_{0}\right)}{2} .
\end{aligned}
$$

Lemma 5.7. There exists $\Psi_{n}: \mathbb{R}_{\geq 0} \longrightarrow \mathbb{R}_{\geq 0} \quad C^{\infty}$ increasing which satisfies the following properties:

(i) $\forall t \in\left[0, \frac{1}{2 n}\right], \Psi_{n}(t)=t+\frac{1}{8 n}$;

(ii) $\forall t \in\left[11 n-\frac{1}{8 n}, \infty\right), \Psi_{n}(t) \geq 11 n+\max \left\{V(x): V_{\alpha_{n}}(x) \leq t\right\}$;

(iii) $\forall t \in\left[\frac{1}{n}-\frac{1}{8 n}, 10 n\right], \Psi_{n}(t)=t$;

(iv) $\forall t \geq 0, \Psi_{n}^{\prime}(t) \geq \frac{1}{2}$.

Proof. The different properties lead to defining a piecewise affine function which we then regularize to render it $C^{\infty}$, giving $\Psi_{n}$.

We now set $\tilde{V}_{n}:=\Psi_{n} \circ V_{\alpha_{n}}$, this function is semiconcave on $\mathbb{X}$ by Lemma 5.1. The definitive Lyapunov pair $(\mathcal{V}, \mathcal{W})$ is defined for all $x \in \mathbb{X}$ by:

$$
\mathcal{V}(x):=\min _{n \in \mathbb{N}^{*}}\left\{\tilde{V}_{n}(x)\right\} \quad \text { and } \mathcal{W}(x):=\frac{W(x)}{4} .
$$

Lemma 5.8. $\forall n \in \mathbb{N}^{*}, \forall x_{0} \in S_{V}\left[\frac{1}{n}, 10 n\right], \mathcal{V}\left(x_{0}\right)=\min _{1 \leq p \leq n} \tilde{V}_{p}\left(x_{0}\right)$. Furthermore, if $\zeta \in \partial_{P} \mathcal{V}\left(x_{0}\right)$, then

$$
\inf _{u \in U}\left\langle\zeta, f\left(x_{0}, u\right)\right\rangle \leq-\frac{W\left(x_{0}\right)}{4} .
$$

Proof. Let be given $n \in \mathbb{N}^{*}$ and $x_{0} \in S_{V}\left[\frac{1}{n}, 10 n\right]$. By Lemma 5.5, $V_{\alpha_{n}}\left(x_{0}\right) \in$ $\left[\frac{1}{n}-\frac{1}{8 n}, 10 n\right]$. Hence, Lemma 5.7 implies that $\tilde{V}_{n}\left(x_{0}\right)=V_{\alpha_{n}}\left(x_{0}\right)$. On the other hand, for any $p \geq n$, by construction $\alpha_{p} \geq \alpha_{n}$ and then $V_{\alpha_{p}}\left(x_{0}\right) \geq$ $V_{\alpha_{n}}\left(x_{0}\right)$. The same argument as above on $\Psi_{p}$ leads to

$$
\tilde{V}_{p}\left(x_{0}\right)=V_{\alpha_{p}}\left(x_{0}\right) \geq \tilde{V}_{n}\left(x_{0}\right)=V_{\alpha_{n}}\left(x_{0}\right) .
$$

consequently, we have shown that $\mathcal{V}\left(x_{0}\right)=\min _{1 \leq p \leq n} \tilde{V}_{p}\left(x_{0}\right)$. Now, if the minimum in the definition of $\mathcal{V}\left(x_{0}\right)$ is attained for $\tilde{\tilde{V}}_{n_{0}}\left(x_{0}\right)$ (with $1 \leq n_{0} \leq n$ ) then

$$
\zeta \in \partial_{P} \mathcal{V}\left(x_{0}\right) \Longrightarrow \zeta \in \partial_{P} \tilde{V}_{n_{0}}\left(x_{0}\right)=\Psi^{\prime}\left(V_{\alpha_{n_{0}}}\left(x_{0}\right)\right) \partial_{P} V_{\alpha_{n_{0}}}\left(x_{0}\right)
$$

We now have to show the inequality (5.5).

First Case: If $V\left(x_{0}\right)>11 n_{0}$ and $V_{\alpha_{n_{0}}\left(x_{0}\right)} \leq 11 n_{0}$, then there exists $\bar{y} \in x_{0}+\bar{B}$ IGD, , , 
(because $\left.\alpha_{n_{0}} \geq 11 n_{0}\right)$ such that $V_{\alpha_{n_{0}}}\left(x_{0}\right)=V(\bar{y})+\alpha_{n_{0}}\left\|x_{0}-\bar{y}\right\|^{2}$. Therefore, $\bar{y} \in S_{V}\left(11 n_{0}\right)$ and $x_{0} \in S_{V}\left(M_{n_{0}}\right)$ by definition of $M_{n_{0}}$. By Lemma 5.5 and Lemma 5.7 (ii), we obtain $V_{\alpha_{n_{0}}\left(x_{0}\right)} \geq 11 n_{0}-\frac{1}{8 n_{0}}$ and $\tilde{V}_{n_{0}}\left(x_{0}\right) \geq 11 n_{0}+V\left(x_{0}\right)$. But $\tilde{V}_{n}\left(x_{0}\right)=V_{\alpha_{n}}\left(x_{0}\right) \leq V\left(x_{0}\right)$. Hence, $n_{0}=n$, and we have the decrease property by Lemma 5.6 .

Second Case: If $V\left(x_{0}\right)>11 n_{0}$ and $V_{\alpha_{n_{0}}\left(x_{0}\right)}>11 n_{0}$, then Lemma 5.7(ii) implies $\tilde{V}_{n_{0}}\left(x_{0}\right) \geq 11 n_{0}+V\left(x_{0}\right)$, we conclude as in the first case.

Third Case: If $V\left(x_{0}\right)<\frac{1}{2 n_{0}}$, then

$$
V_{\alpha_{n_{0}}}\left(x_{0}\right) \leq V\left(x_{0}\right)<\frac{1}{2 n_{0}} \Longrightarrow \tilde{V}_{n_{0}}\left(x_{0}\right)=V_{\alpha_{n_{0}}}\left(x_{0}\right)+\frac{1}{8 n_{0}} \geq V\left(x_{0}\right) .
$$

But we proved that $\tilde{V}_{n}\left(x_{0}\right)=V_{\alpha_{n}}\left(x_{0}\right) \leq V\left(x_{0}\right)$, so the minimum is also attained for $n$; then we have (5.5) by Lemma 5.6.

Fourth Case: If $x_{0} \in S_{V}\left[\frac{1}{2 n_{0}}, 11 n_{0}\right]$, then we conclude by Lemma 5.6 and Lemma 5.7 (iv).

This last Lemma shows that the minimum in the definition of $\mathcal{V}(x)$ is always attained for $x \neq 0$. Therefore, the function $\mathcal{V}$ is semiconcave outside the origin (by Lemma 5.2). On the other hand, $\mathcal{V}$ is continuous on the origin (because $0 \leq \mathcal{V} \leq V$ ) and satisfies the decrease condition by 5.5 . Consequently $\mathcal{V}$ provides a control-Lyapunov function; which proves the Theorem 2.10.

Acknowledgments. I would like to thank Professor Francis Clarke for his comments and his suggestions.

\section{REFERENCES}

[1] G. Alberti, L. Ambrosio, and P. Cannarsa. On the singularities of convex functions. Manuscripta Math., 76(3-4):421-435, 1992.

[2] L. Ambrosio, P. Cannarsa, and H. M. Soner. On the propagation of singularities of semi-convex functions. Ann. Scuola Norm. Sup. Pisa Cl. Sci. (4), 20(4):597-616, 1993.

[3] Z. Artstein. Stabilization with relaxed controls. Nonlinear Analysis TMA, 7:1163$1173,1983$.

[4] J.P. Aubin and A. Cellina. Differential Inclusions. Springer-Verlag, 1984.

[5] A. Bacciotti. Local Stabilizability of Nonlinear Control Systems. World Scientific, 1992.

[6] A. Bacciotti and L. Rosier. Lyapunov and Lagrange stability: inverse theorems for discontinuous systems. Math. Control Signals Systems, 11:101-128, 1998.

[7] M. Bardi and I. Capuzzo-Dolcetta. Optimal control and viscosity solutions of Hamilton-Jacobi-Bellman equations. Birkhäuser Boston Inc., Boston, MA, 1997.

[8] R.W. Brockett. Asymptotic stability and feedback stabilization. In R.W. Brockett, R.S. Millman, and H.J. Sussmann, editors, Differential Geometric Control Theory, pages 181-191. Birkhäuser, Boston, 1983.

[9] P. Cannarsa and C. Sinestrari. Convexity properties of the minimum time function. Calc. Var. Partial Differential Equations, 3(3):273-298, 1995.

[10] F.H. Clarke, Yu.S. Ledyaev, L. Rifford, and R.J. Stern. Feedback stabilization and Lyapunov functions. To appear.

[11] F.H. Clarke, Yu.S. Ledyaev, E.D. Sontag, and A.I. Subbotin. Asymptotic controllability implies feedback stabilization. I.E.E.E. Trans. Aut. Control, 42:1394-1407, 1997.

[12] F.H. Clarke, Yu.S. Ledyaev, and R.J. Stern. Asymptotic stability and smooth Lyapunov functions. J. Differential Equations, 149:69-114, 1998. 
[13] F.H. Clarke, Yu.S. Ledyaev, R.J. Stern, and P.R. Wolenski. Qualitative properties of trajectories of control systems: a survey. J. Dyn. Control Sys., 1:1-48, 1995.

[14] F.H. Clarke, Yu.S. Ledyaev, R.J. Stern, and P.R. Wolenski. Nonsmooth Analysis and Control Theory. Graduate Texts in Mathematics, vol. 178. Springer-Verlag, New York, 1998.

[15] J-M. Coron. Global asymptotic stabilization for controllable systems without drift. Math. Cont. Sig. Sys., 5:295-312, 1992.

[16] J-M. Coron. Stabilization in finite time of locally controllable systems by means of continuous time-varying feedback laws. SIAM J. Control and Optim., 33:804-833, 1995.

[17] R. Freeman and P.V. Kokotovic. Robust Nonlinear Control Design. State-Space and Lyapunov Techniques. Birkhäuser, 1996.

[18] H. Hermes. Resonance, stabilizing feedback controls, and regularity of viscosity solutions of Hamilton-Jacobi-Bellman equations. Math. Control Signals Systems, 9:59-72, 1996.

[19] S. N. Kružkov. Generalized solutions of Hamilton-Jacobi equations of eikonal type I. Math. USSR-Sb, 27:406-446, 1975.

[20] P-L. Lions. Generalized solutions of Hamilton-Jacobi equations. Pitman (Advanced Publishing Program), Boston, Mass., 1982.

[21] L. Rosier. Étude de quelques problèmes de stabilisation. PhD thesis, ENS de Cachan, 1993.

[22] E.P. Ryan. On Brockett's condition for smooth stabilizability and its necessity in a context of nonsmooth feedback. S.I.A.M. J. Control Optim., 32:1597-1604, 1994.

[23] E.D. Sontag. A Lyapunov-like characterization of asymptotic controllability. SIAM J. Control and Optim., 21:462-471, 1983.

[24] E.D. Sontag and H.J. Sussmann. Remarks on continuous feedback. In Proc. I.E.E.E. Conf. Decision and Control, Albuquerque, pages 916-921, Piscataway, 1980. IEEE Publications.

[25] E.D. Sontag and H.J. Sussmann. Nonsmooth control-Lyapunov functions. In Proc. I.E.E.E. Conf. Decision and Control, New Orleans. IEEE Publications, 1995.

[26] E.D. Sontag and H.J. Sussmann. General classes of control-Lyapunov functions. In Stability theory (Ascona, 1995), pages 87-96. Birkhäuser, Basel, 1996. 